\title{
Predicting Infection Risk in Multiple Sclerosis Patients Treated with Ocrelizumab: A Retrospective Cohort Study
}

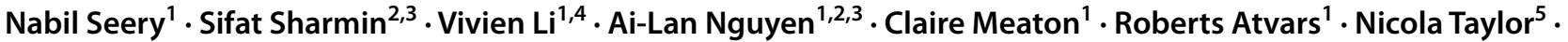 \\ Kelsey Tunnell ${ }^{5}$. John Carey ${ }^{5}$. Mark P. Marriott ${ }^{1,6} \cdot$ Katherine A. Buzzard $^{1,6} \cdot$ Izanne Roos $^{1,2,3}$. Chris Dwyer ${ }^{1,4}$. \\ Josephine Baker ${ }^{1} \cdot$ Lisa Taylor $^{1} \cdot$ Kymble Spriggs $^{3,7} \cdot$ Trevor J. Kilpatrick $^{1,4} \cdot$ Tomas Kalincik $^{1,2,3} \cdot$ Mastura Monif $^{1,8,9}$
}

Accepted: 25 March 2021 / Published online: 13 April 2021

(c) The Author(s), under exclusive licence to Springer Nature Switzerland AG 2021

\begin{abstract}
Background Ocrelizumab safety outcomes have been well evaluated in clinical trials and open-label extension (OLE) studies. However, risk factors for infection in patients with multiple sclerosis (MS) receiving ocrelizumab have not been extensively studied in the real-world setting.

Objective The aim of this study was to examine factors determining risk of self-reported infections and antimicrobial use in patients receiving ocrelizumab for MS.

Methods A retrospective, observational cohort study was conducted in patients receiving ocrelizumab at the Royal Melbourne Hospital. Infection type and number were reported by patients, and the associations of potential clinical and laboratory risk factors with self-reported infection and antimicrobial use were estimated using univariate and multivariable logistic regression models.

Results A total of 185 patients were included in the study; a total of 176 infections were reported in 89 patients (46.1\%), and antimicrobial use was identified in 47 patients (25.3\%). In univariate analyses, a higher serum IgA was associated with reduced odds of infection (OR 0.44, 95\% CI 0.25-0.76). In multivariable analyses, older age (OR 0.94, 95\% CI 0.88-0.99), higher serum IgA (OR 0.37, 95\% CI 0.17-0.80) and higher serum IgG (OR 0.81, 95\% CI 0.67-0.99) were associated with reduced odds of infection. Older age (OR 0.85, 95\% CI 0.75-0.96) and higher serum IgA (OR 0.23, 95\% CI 0.07-0.79) were associated with reduced odds of antimicrobial use, whilst longer MS disease duration (OR 1.22, 95\% CI 1.06-1.41) and higher Expanded Disability Status Scale (EDSS) score (OR 1.99, 95\% CI 1.02-3.86) were associated with increased odds of antimicrobial use.

Conclusions Higher serum IgA and IgG and older age were associated with reduced odds of infection. Our findings highlight that infection risk is not uniform in patients with MS receiving ocrelizumab and substantiate the need to monitor immunoglobulin levels pre-treatment and whilst on therapy.
\end{abstract}

\section{Introduction}

Multiple sclerosis (MS) is a chronic inflammatory demyelinating disorder of the central nervous system, and a foremost cause of disability in young adults. Highly effective disease modifying therapies (DMTs) in relapsing-remitting MS (RRMS) have shown superior efficacy in reducing the rate of clinical relapses, MRI activity and disability accumulation in randomised clinical trials (RCTs). Observational data has shown that initial treatment with higher efficacy DMTs is

Mastura Monif

mastura.monif@mh.org.au

Extended author information available on the last page of the article associated with a more favourable long-term Expanded Disability Status Scale (EDSS) score [1,2] and a lower risk of conversion to secondary-progressive MS (SPMS) [3]. Use of higher efficacy DMTs is chiefly balanced against increased risk of serious adverse events, particularly risk of infection. Compared with the general population, patients with MS have been shown to have higher rates of infection [4] and infection-related health-care utilisation, including hospitalisation [4, 5], and are more likely to die from infectious causes [6-8]. DMTs appear to be associated with varying infection risk, with real-world data indicating a higher risk of infection-related hospitalisation for fingolimod, natalizumab and rituximab compared with both the general population and MS patients treated with injectable therapies [9]. 


\section{Key Points}

In a retrospective cohort of 185 patients with multiple sclerosis treated with ocrelizumab, 176 infections were reported, in $46.1 \%$ of patients.

Odds of infection in univariate and multivariable analyses were not uniform, with increasing IgA and IgG and multiple clinical factors being associated with reduced odds of infection.

Also, higher rates of infections were seen in MS patients on natalizumab and fingolimod compared with MS patients not exposed to DMTs [10].

While MS is traditionally thought to be a T-cell-mediated disease, B-cells are now recognised as integral to the pathophysiology of MS, as evidenced by (1) intrathecal oligoclonal band synthesis, (2) B-cell detection in meningeal aggregates and leptomeningeal lymphoid follicles, (3) B-cells in perivascular lesions, and (4) the high efficacy of B-cell depleting therapies $[11,12]$, as demonstrated in the pivotal phase II and III studies in RRMS [13-15] and primary progressive MS (PPMS) [16].

In phase III randomised trials, ocrelizumab, a fully humanised anti-CD20 monoclonal antibody, was not associated with higher rates of serious infections compared with interferon- $\beta$ in RRMS [14] and placebo in PPMS [16]. Higher rates of upper respiratory tract infection and herpes infections, however, were seen in ocrelizumab-treated patients in both studies. Further, a higher rate of nasopharyngitis was observed in ocrelizumab-treated patients compared with interferon- $\beta$ in the RRMS groups $[14,16]$. On the other hand, use of ocrelizumab in rheumatoid arthritis in combination with methotrexate resulted in an increased rate of serious infections compared with methotrexate use alone [17]. Emerging data has indicated a possible association between B-cell depleting therapies and more severe COVID-19 outcomes [18, 19], engendering clinical uncertainty with regards to initiating, continuing or postponing treatment with B-cell depleting therapies in patients with MS.

Identifying patients who may be at higher risk of infection when treated with ocrelizumab and other B-cell depleting DMTs is therefore an important consideration. The primary aim of this study was to identify risk factors for infection of any kind in patients with MS treated with ocrelizumab in a tertiary health centre in Victoria, Australia.

\section{Methods}

\subsection{Study Design}

In a single-centre, retrospective, observational cohort study of patients with MS treated with ocrelizumab, from 1 April until 2 October 2020, we evaluated the number of infections reported by patients since their prior infusion, either via voluntary survey completed by patients upon attending hospital for their ocrelizumab infusions, or via a phone survey following informed consent. The study received ethics approval from The Royal Melbourne Hospital Human Research Ethics Committee (QA2020045).

Patients were asked to note if they had any upper or lower respiratory tract infections, flu-like illness, herpes mucocutaneous lesions (including herpes zoster), urinary tract infections, gastrointestinal infection, cellulitis, or other infections, by either selecting pre-defined descriptions of symptoms from a list, or detailing their infective symptoms (see supplementary appendix in the electronic supplementary material $[\mathrm{ESM}]$ ), also listing the number of such infections to a maximum of four for a given type, except herpes labialis, for which the maximum number in a given patient was counted as one, given such infections were deemed most likely to be prone to recall bias. They were also asked if antimicrobial use was initiated for a given infection. The reported interval of interest was the time between the most recent two infusions of ocrelizumab (or the time of completing a phone survey where an infusion was deferred).

Further, patients were asked to report if their rate of infection had remained unaltered, increased or decreased since commencing ocrelizumab, and to report influenza vaccination administered since their last infusion. All patients were also asked about smoking status.

Where possible, external general practice records (from their primary care physician) were obtained to confirm infection-related health-care consultations and antimicrobial utilisation pertaining to infections outlined by patients in their survey, and hospital records were used to identify any admissions due to infection in the corresponding time period, as well as potential infusion-related reactions (IRR) that could confound interpretation of relevant infections as reported by patients.

\subsection{Data Acquisition}

Variables identified for analysis as potential contributors to infection risk included age, sex, MS duration, EDSS, number of ocrelizumab cycles and prior immunosuppression (denoting any prior DMT other than interferon- $\beta$, glatiramer acetate and intravenous immunoglobulin [IVIg]), IgG, IgA and IgM levels, total lymphocyte levels, neutrophil counts and 
selected lymphocyte subsets. In parallel to evaluating risk of infection, risk of antimicrobial use was elaborated. A composite variable of self-reported or confirmed antimicrobial use was developed to approximate the proportion of patients sustaining infections deemed to require antimicrobials. In addition to patients self-reporting antimicrobial use, a proportion of patients not reporting antimicrobial use reported a health-care visit for an infection, and where subsequent review of relevant medical records revealed prescription of an antimicrobial agent, such cases were included in the composite variable. This was thought overall to reflect a more accurate representation of the proportion of patients prescribed antimicrobials.

Baseline demographic data was obtained from iMed, an MS clinical data entry system. Electronic medical records and laboratory data on white cell count differentials, lymphocyte subsets and immunoglobulin levels were obtained from the Royal Melbourne Hospital electronic medical records system and external pathology electronic records. Lymphocyte subsets were measured and quantified as follows: total lymphocyte count and absolute counts of $\mathrm{CD}^{+}$, $\mathrm{CD}^{+}, \mathrm{CD}^{+}$, and $\mathrm{CD} 19^{+}$.

\subsection{Statistical Analysis}

Statistical analysis was carried out using R version 1.2.5033. Means and standard deviations (SDs) were obtained where appropriate to summarise demographic clinical and laboratory data. Logistic regression models within the generalised linear model framework were used to perform univariate and multivariable analyses, to estimate the associations of selected clinical and laboratory variables with the outcomes of self-reported infection and self-reported or confirmed antimicrobial use. Accordingly, odds ratios (ORs) and 95\% confidence intervals $(95 \% \mathrm{CI}$ ) were calculated. Variables selected for multivariable analyses included those with $p<0.05$ in the univariate analyses, and variables deemed important clinical confounders of infection risk, specifically, age, gender, EDSS score, disease duration, number of ocrelizumab cycles and respective immunoglobulin levels.

To account for the effect of the variability in the reported interval for infections, the logarithm of the time from a patient's prior infusion until the time of the survey was included as an offset in each of the logistic regression models.

The timing of pre-infusion lymphocyte and immunoglobulin measurements was subject to variation. Given the propensity for reconstitution of lymphocyte and immunoglobulin levels with time since $\mathrm{CD} 20^{+}$depletion, a binary correction variable ( 1 for patients who had a blood test after the median time interval since their prior infusion [5.8 months] and 0 otherwise) was included in all the models involving a laboratory variable.
Immunoglobulin data were only available for subgroups of the study cohort. Therefore, a multivariable model was run separately for $\operatorname{IgG}, \operatorname{IgM}$ and $\operatorname{Ig} \mathrm{A}$ within these respective subgroups.

\section{Results}

\subsection{Demographic Results}

Of 237 patients receiving infusions from April 1, 2020 until October 12, 2020, 207 completed the survey. Of these, 22 patients who were receiving their first dose of ocrelizumab were excluded. In total, 185 patients with MS were included in the study, with 126 completing the survey individually at the time of their most recent infusion, and a further 59 patients completing the survey via phone. Demographics and clinical characteristics are outlined in Table 1.

In brief, the mean (SD) age was 43.4 (10.6) years, 134 of $185(72.4 \%)$ were female, the mean (SD) disease duration was 10.7 (7.5) years, 50 (29.4\%) patients were DMTnaïve prior to ocrelizumab, the median (1st, 3rd quartiles) EDSS score was $2.0(1,4)$ and the mean $(\mathrm{SD})$ number of ocrelizumab doses was 4.6 (1.3). One hundred patients who had transitioned off a prior DMT had washout information available. The number of patients with available intervals and the median washout (range) for respective DMTs were as follows: natalizumab: 52 patients, 42.5 (16-446) days; fingolimod: 32 patients, 41.0 (16-125) days; rituximab: 11 patients, 189.0 (117-227) days; dimethyl fumarate: 2 patients, 23.5 (8-39) days; alemtuzumab: 2 patients, 742.5 (604-881) days; and daclizumab: 1 patient, 33.0 days.

Immunoglobulin levels for analysis were available as follows: 131 patients had IgG levels, 98 had IgA levels and 107 had available IgM levels. Of patients reporting infections, available immunoglobulin levels were as follows: 68 of $89(76 \%)$ for $\operatorname{IgG}, 65(73 \%)$ for $\operatorname{IgA}$ and $66(74 \%)$ for IgM. Mean immunoglobulin levels of $\operatorname{IgG}, \operatorname{IgA}$ and $\operatorname{IgM}$ were $9.54 \pm 2.40 \mathrm{~g} / \mathrm{L}, 1.80 \pm 0.92 \mathrm{~g} / \mathrm{L}$ and $0.70 \pm 0.48 \mathrm{~g} / \mathrm{L}$, respectively, with $2.3 \%, 3.1 \%$ and $9.3 \%$ of patients, respectively, recording levels below the lower limit of normal prior to their index infusion. The mean lymphocyte count was $1.67 \pm 0.70 \times 10^{9} / \mathrm{L}$, mean neutrophil count was $4.57 \pm 1.92$ $\times 10^{9} / \mathrm{L}$ and mean $\mathrm{CD} 4^{+}, \mathrm{CD}^{+}$and $\mathrm{CD} 19^{+}$counts were $0.93 \pm 0.51 \times 10^{9} / \mathrm{L}, 0.40 \pm 0.27 \times 10^{9} / \mathrm{L}$ and $0.02 \pm 0.06$ $\times 10^{9} / \mathrm{L}$, respectively.

\subsection{Infections, Health-Care Utilisation, Hospitalisation}

The total number of self-reported infections was 176 , in 89 (48.1\%) patients. Upper respiratory tract infections ( 78 total in $27.6 \%$ of patients) were the most common, followed by 
Table 1 Demographics and clinical characteristics

Total number of patients with MS

185

Self-filled survey, $N(\%)$

$126(68.1)$

Phone survey, $N(\%)$

59 (31.9)

Age, years (mean $\pm \mathrm{SD}$ )

$43.4 \pm 10.6$

Female sex, $N(\%)$

$134(72.4)$

Type of multiple sclerosis, $N(\%)$

Relapsing-remitting

Secondary progressive

$7(3.8)$

Primary progressive

$7(3.8)$

MS/MOGAD overlap

$4(2.2)$

Disease duration, years (mean $\pm \mathrm{SD}$ )

$10.65 \pm 7.46$

Currently smoking, $N(\%)$

Influenza vaccination 2020, $N(\%)$

$97(52.4)$

Pneumococcal vaccination 2020, $N(\%)$

$4(2.2)$

No prior DMT, $N(\%)$

$50(29.4)$

DMT immediately prior to ocrelizumab, $N\left(\%^{\mathrm{a}}\right)$, [median washout, days]

117

Any interferon beta

4 (3.4)

Glatiramer acetate

$5(4.3)$

Dimethyl fumarate

7 (6.0), [23.5]

Fingolimod

34 (29.0), [41.0]

Natalizumab

52 (44.0), [42.5]

Alemtuzumab

2 (1.7), [742.5]

Rituximab

$11(9.4),[189]$

Other

$2(1.7)$

Median EDSS score, (1st, 3rd quartiles)

$2.0(1,4)$

Ocrelizumab doses, (mean \pm SD)

$4.6 \pm 1.3$

Mean study period interval, months, (mean \pm SD)

$6.7 \pm 1.5$

Median time from prior infusion to lymphocyte, immunoglobulin levels, months, (1st, 3rd quartiles)

$5.8(5.4,6.0)$

Total patient-years of exposure

103.9

$\mathrm{IgG}, \mathrm{g} / \mathrm{L}$, mean $\pm \mathrm{SD},(N)$

$9.54 \pm 2.40(131)$

$\mathrm{IgG}<\mathrm{LLN}^{\mathrm{b}}, N(\%)$

$3(2.3)$

$\mathrm{IgA}, \mathrm{g} / \mathrm{L}$, mean $\pm \mathrm{SD},(N)$

$1.80 \pm 0.92(98)$

$\operatorname{IgA}<\mathrm{LLN}^{\mathrm{b}}, N(\%)$

$3(3.1)$

$\mathrm{IgM}, \mathrm{g} / \mathrm{L}$, mean $\pm \mathrm{SD},(N)$

$0.70 \pm 0.48(107)$

$\operatorname{IgM}<\operatorname{LLN}^{\mathrm{b}}, N(\%)$

$10(9.3)$

Lymphocyte count $\times 10^{9} / \mathrm{L}$, mean $\pm \mathrm{SD},(N)$

$1.67 \pm 0.70(168)$

Neutrophil count $\times 10^{9} / \mathrm{L}$, mean $\pm \mathrm{SD},(N)$

$4.57 \pm 1.92(165)$

$\mathrm{CD} 4^{+}$count $\times 10^{9} / \mathrm{L}$, mean $\pm \mathrm{SD},(N)^{\mathrm{c}}$

$0.93 \pm 0.51(85)$

$\mathrm{CD} 8^{+}$count $\times 10^{9} / \mathrm{L}$, mean $\pm \mathrm{SD},(N)^{\mathrm{c}}$

$0.40 \pm 0.27(85)$

$\mathrm{CD} 19^{+}$count $\times 10^{9} / \mathrm{L}$, mean $\pm \mathrm{SD},(N)^{\mathrm{c}}$

$0.02 \pm 0.06(86)$

$D M T$ disease-modifying therapy, EDSS Expanded Disability Status Scale, ESM electronic supplementary material, $L L N$ lower limit of normal, $M O G A D$ myelin-oligodendrocyte antibody disorders, $M S$ multiple sclerosis, $S D$ standard deviation

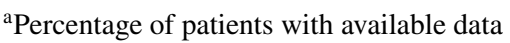

${ }^{\mathrm{b}}$ Includes patients who had lymphocyte subsets at extended intervals

cPlease see ESM for LLN reference ranges

urinary tract infections (33 total in $11.9 \%$ of patients). No patients contracted SARS-CoV-2. Further details on the type of reported infections and frequency are outlined in Table 2. The rate of self-reported infections was 169.4 per 100 patient years (PY). Of these, 47 patients reported antimicrobial prescription and 27 had confirmed infection-related health-care visits, totalling 45 visits, with prescription of antimicrobials confirmed on 39 occasions, with a rate of prescription of 
37.5 per 100 PY. Five (2.7\%) patients were hospitalised, with hospital diagnoses of a viral infection with superimposed bacterial infection, osteomyelitis, urosepsis, cystitis and pyelonephritis. Details as to the clinical characteristics of these patients can be found in the ESM. The majority of patients $(72 \%)$ reported no change in the frequency of infection since commencing ocrelizumab, with $16 \%$ perceiving an increased frequency and $12 \%$ a decreased frequency.

On review of medical records in the relevant reporting period, in patients reporting respiratory symptoms, documentation of a likely IRR was confirmed in one patient contemporaneous with their infusion (feeling flushed, noted to be tachycardic), but in this patient an upper respiratory infection was confirmed via external health records corresponding to a distant future date. Three other patients reported symptoms during their ocrelizumab infusions, thought unlikely to confound interpretation of descriptions of subsequent infections - in brief, self-limiting dizziness in one patient, and worsening fatigue in two other patients.

Table 2 Self-reported infections

\begin{tabular}{ll}
\hline Total number of infections & 176 \\
Total infection per 100 PY & 169.4 \\
Total number patients reporting infection (\%) & $89(48.1)$ \\
Total patients prescribed antimicrobials (\%) & $47(25.3)$ \\
Total number of grade 2 and 3 infections & 63 \\
Total number of grade 3 infections ${ }^{\mathrm{a}}(\%)$ & $5(2.7)$ \\
Hospitalisation rate per 100 PY & 4.8 \\
Confirmed total health-care visits & 45 \\
Confirmed patients with health-care visits & 27 \\
Health-care visits per 100 PY & 43.3 \\
Confirmed antimicrobial prescriptions & 39 \\
Confirmed antimicrobial prescriptions per 100 PY & 37.5 \\
Self-reported infection, $N(\%)$ & \\
Upper respiratory tract infection & $78(27.6)$ \\
Influenza-like illness & $9(3.2)$ \\
Lower respiratory tract infection & $7(2.7)$ \\
Urinary tract infection & $33(11.9)$ \\
Skin infection, bacterial & $6(2.7)$ \\
Skin infection, viral & $4(1.1)$ \\
Herpes labialis, mouth or genital ulcers & $14(7.6)$ \\
Gastroenteritis & $14(5.9)$ \\
Other & $11(5.4)$ \\
Self-reported change in infection rate, $N(\%)$ & \\
No change & $125(72)$ \\
Increase & $28(16)$ \\
Decrease & $21(12)$ \\
\hline
\end{tabular}

$P Y$ patient years

${ }^{a}$ Hospital diagnoses were osteomyelitis (1), viral and superimposed bacterial infection (1), urosepsis (1), cystitis (1), pyelonephritis (1)

\subsection{Univariate, Multivariable Analyses of Predictors of Infection and Antimicrobial Use}

In the univariate analyses (Table 3), only higher serum IgA showed a statistically significant association with decreased odds of infection (OR 0.44, 95\% CI 0.25-0.76). In a clinical multivariable model ( $n=139$, Table 4$)$, we did not find evidence for independent associations between any of age, gender, disease duration, doses of ocrelizumab and EDSS score and self-reported infection risk. In a separate multivariable model, higher IgA levels $(n=73)$ were associated with lower odds of infection (OR $0.37,95 \%$ CI $0.17-0.80$ ). In a multivariable model evaluating $\operatorname{IgG}$ and the prior clinical variables $(n=104)$, older age (OR $0.94,95 \%$ CI $0.89-0.99)$, and higher serum IgG (OR 0.81, 95\% CI 0.67-0.99) were associated with lower odds of infection. Finally, in a multivariable model including IgM levels $(n=83$, Table 4$)$, only older age was associated with lower odds of infection (OR $0.93,95 \%$ CI $0.88-0.99$ ).

The univariate analyses (Table 5) did not identify any variables showing a significant association with use of antimicrobial therapy. In a clinical multivariable model, only older age was associated with lower odds of antimicrobial therapy use (OR $0.95,95 \%$ CI $0.91-1.00$ ). In three further multivariable models (Table 6), older age and shorter disease duration were associated with a lower risk of using antimicrobial therapy. In one model, lower EDSS (OR 1.99, 95\% CI 1.02-3.86) and higher IgA levels (OR 0.23 , 95\% CI 0.07-0.79) were associated with lower odds of antimicrobial

Table 3 Univariate analysis of the predictors of self-reported infection

\begin{tabular}{lll}
\hline Variable & OR $(95 \%$ CI $)$ & $p$ value \\
\hline Age & $0.97(0.95-1.00)$ & 0.06 \\
Gender, female & $1.85(0.96-3.65)$ & 0.07 \\
Disease duration & $0.98(0.94-1.02)$ & 0.36 \\
Number of ocrelizumab doses & $0.91(0.72-1.14)$ & 0.41 \\
EDSS & $0.92(0.78-1.08)$ & 0.30 \\
Prior immunosuppression & $1.63(0.83-3.17)$ & 0.15 \\
Lymphocyte count & $1.37(0.88-2.13)$ & 0.17 \\
Neutrophil count & $1.10(0.93-1.29)$ & 0.28 \\
IgG level & $0.89(0.77-1.04)$ & 0.15 \\
IgA level & $\mathbf{0 . 4 4}(\mathbf{0 . 2 5 - 0 . 7 6 )}$ & $\mathbf{0 . 0 0 4}$ \\
IgM level & $0.62(0.26-1.49)$ & 0.29 \\
$\mathrm{CD}^{+}$ & $1.42(0.52-3.89)$ & 0.49 \\
$\mathrm{CD}^{+}$ & $0.36(0.06-2.31)$ & 0.28 \\
$\mathrm{CD}^{+} 9^{+}$ & $3.01(0.00-4986.26)$ & 0.77 \\
\hline
\end{tabular}

Self-reported infection included any patient that reported at least one infection or more. Bold values indicate statistical significance

$C I$ confidence intervals, EDSS Expanded Disability Status Scale, $O R$ odds ratio 
Table 4 Multivariable analyses of the predictors of self-reported infection.

\begin{tabular}{|c|c|c|c|c|c|c|c|c|}
\hline \multirow[t]{2}{*}{ Variable } & \multicolumn{2}{|c|}{ Clinical model $(N=139)$} & \multicolumn{2}{|c|}{$\begin{array}{l}\text { Clinical model with IgA }(N \\
=73)\end{array}$} & \multicolumn{2}{|c|}{$\begin{array}{l}\text { Clinical model with IgG }(N \\
=104)\end{array}$} & \multicolumn{2}{|c|}{$\begin{array}{l}\text { Clinical model with } \operatorname{IgM}(N \\
=83)\end{array}$} \\
\hline & OR $(95 \% \mathrm{CI})$ & $p$ value & OR (95\% CI) & $p$ value & OR $(95 \% \mathrm{CI})$ & $p$ value & OR $(95 \% \mathrm{CI})$ & $p$ value \\
\hline Age & $0.96(0.93-1.00)$ & 0.06 & $0.94(0.88-1.01)$ & 0.07 & $0.94(0.89-0.99)$ & 0.02 & $0.93(0.88-0.99)$ & 0.02 \\
\hline Female gender & $2.00(0.93-4.30)$ & 0.08 & $2.48(0.73-8.41)$ & 0.14 & $2.27(0.91-5.71)$ & 0.08 & $2.27(0.75-6.83)$ & 0.15 \\
\hline Disease duration & $1.00(0.95-1.06)$ & 0.98 & $1.09(1.00-1.18)$ & 0.058 & $1.04(0.98-1.12)$ & 0.21 & $1.05(0.98-1.14)$ & 0.18 \\
\hline $\begin{array}{l}\text { Number of ocreli- } \\
\text { zumab doses }\end{array}$ & $0.97(0.73-1.28)$ & 0.82 & $0.92(0.56-1.52)$ & 0.76 & $0.96(0.67-1.38)$ & 0.84 & $1.01(0.66-1.55)$ & 0.97 \\
\hline EDSS & $0.98(0.81-1.18)$ & 0.80 & $1.09(0.75-1.60)$ & 0.66 & $0.96(0.74-1.25)$ & 0.75 & $1.11(0.80-1.54)$ & 0.52 \\
\hline IgA level & & & $0.37(0.17-0.80)$ & 0.01 & & & & \\
\hline IgG level & & & & & $0.81(0.67-0.99)$ & 0.04 & & \\
\hline IgM level & & & & & & & $0.56(0.19-1.67)$ & 0.30 \\
\hline
\end{tabular}

Bold values indicate statistical significance

CI confidence intervals, EDSS Expanded Disability Status Scale, OR odds ratio

Table 5 Univariate analysis of the predictors of self-reported and confirmed antimicrobial use

\begin{tabular}{lll}
\hline Variable & OR $(95 \%$ CI $)$ & $p$ value \\
\hline Age & $0.98(0.96-1.02)$ & 0.43 \\
Gender, female & $2.30(0.99-5.36)$ & 0.05 \\
Disease duration & $1.02(0.97-1.07)$ & 0.53 \\
Number of ocrelizumab doses & $0.83(0.64-1.08)$ & 0.16 \\
EDSS & $1.16(0.96-1.39)$ & 0.12 \\
Prior immunosuppression & $1.77(0.80-3.95)$ & 0.16 \\
Lymphocyte count & $1.51(0.93-2.50)$ & 0.10 \\
Neutrophil count & $1.12(0.94-1.34)$ & 0.22 \\
IgG level & $0.87(0.72-1.06)$ & 0.16 \\
IgA level & $0.54(0.28-1.05)$ & 0.07 \\
IgM level & $0.22(0.04-1.10)$ & 0.07 \\
CD4 ${ }^{+}$ & $1.15(0.37-3.60)$ & 0.81 \\
$\mathrm{CD}^{+}$ & $0.92(0.12-6.99)$ & 0.94 \\
$\mathrm{CD} 19^{+}$ & $0.09(0.00-7824.67)$ & 0.68 \\
\hline
\end{tabular}

$C I$ confidence intervals, EDSS Expanded Disability Status Scale, $O R$ odds ratio

use $(n=73)$. We did not find evidence for associations of IgG levels ( $n=104$, OR 0.82 , 95\% CI 0.65-1.04) and IgM levels $(n=83$, OR $0.12,95 \%$ CI $0.10-1.50)$ with antimicrobial use.

\section{Discussion}

This study demonstrated that, in multivariable models, higher serum IgA and IgG and younger age were associated with reduced odds of infection. Further, younger age, longer MS disease duration and increased EDSS were associated with increased odds of antimicrobial use, whilst higher serum IgA was associated with reduced odds of antimicrobial use. Our study builds on literature detailing safety outcomes of a B-cell depleting therapy, ocrelizumab, whilst revealing novel factors that could predispose to heightened infection risk and increased infection-related treatment.

The overall rate of self-reported infections in our study of 169.4 per $100 \mathrm{PY}$ was higher than the reported rate of infections in recently published 5-year data from the OPERA double-blind and open-label extension (OLE) studies in RRMS patients [20]. In our study, $16 \%$ of patients were $>$ 55 years of age, and $11 \%$ of patients had an EDSS score $>$ 5.5 , reflecting the greater clinical heterogeneity encountered in a real-world setting. Whilst a direct comparison to the aforementioned findings is not possible given the different methods of defining infections, our findings highlight the possibility of higher rates of infection than seen in selected clinical trial populations. Notwithstanding, older age in our cohort was not associated with increased risk of infection.

The most commonly reported infection in our cohort was that involving the upper respiratory tract ( 78 cases in $27.6 \%$ of patients), followed by urinary tract infection (33 cases in $11.9 \%$ of patients), herpes labialis, mouth or genital ulcers (14 cases in $7.6 \%$ of patients). Review of relevant medical records failed to identify significant IRRs in patients in whom dates of respiratory infections were unknown, thereby potentially confounding interpretation of relevant reported symptoms, though it should be noted that after hospital discharge following an infusion, medical records would only reflect IRRs in patients who had reported attributable symptoms. The ensuing rate of healthcare-related visits that could be confirmed was 43.3 per $100 \mathrm{PY}$, and the rate of confirmed antimicrobial use alone was 37.5 per $100 \mathrm{PY}$. With the exception of two prescriptions of famciclovir for cutaneous herpes simplex virus (HSV), antimicrobials were exclusively represented by antibiotic prescription (a full list 
Table 6 Multivariable analyses of the predictors of self-reported and confirmed antimicrobial use

\begin{tabular}{|c|c|c|c|c|c|c|c|c|}
\hline \multirow[t]{2}{*}{ Variable } & \multicolumn{2}{|c|}{ Clinical model $(N=139)$} & \multicolumn{2}{|c|}{$\begin{array}{l}\text { Clinical model with IgA ( } N \\
=73)\end{array}$} & \multicolumn{2}{|c|}{$\begin{array}{l}\text { Clinical model with } \operatorname{IgG}(N \\
=104)\end{array}$} & \multicolumn{2}{|c|}{$\begin{array}{l}\text { Clinical model with } \operatorname{IgM}(N \\
=83)\end{array}$} \\
\hline & OR $(95 \%$ CI $)$ & $p$ value & OR $(95 \% \mathrm{CI})$ & $p$ value & OR $(95 \%$ CI $)$ & $p$ value & OR $(95 \%$ CI $)$ & $p$ value \\
\hline Age & $0.95(0.91-1.00)$ & 0.048 & $0.85(0.75-0.96)$ & 0.01 & $0.90(0.84-0.97)$ & 0.004 & $0.87(0.78-0.96)$ & 0.007 \\
\hline Female gender & $1.82(0.70-4.77)$ & 0.22 & $3.03(0.32-2.83)$ & 0.33 & $1.27(0.36-4.48)$ & 0.71 & $3.38(0.50-22.82)$ & 0.21 \\
\hline Disease duration & $1.02(0.96-1.09)$ & 0.55 & $1.22(1.06-1.41)$ & 0.006 & $1.10(1.01-1.21)$ & 0.02 & $1.14(1.02-1.28)$ & 0.02 \\
\hline $\begin{array}{l}\text { Number of ocreli- } \\
\text { zumab doses }\end{array}$ & $0.88(0.64-1.22)$ & 0.44 & $0.58(0.27-1.24)$ & 0.16 & $0.85(0.54-1.35)$ & 0.49 & $0.76(0.42-1.37)$ & 0.36 \\
\hline EDSS & $1.21(0.97-1.51)$ & 0.09 & $1.99(1.02-3.86)$ & 0.04 & $1.34(0.95-1.90)$ & 0.10 & $1.46(0.92-2.31)$ & 0.10 \\
\hline IgA level & & & $0.23(0.07-0.79)$ & 0.02 & & & & \\
\hline IgG level & & & & & $0.82(0.65-1.04)$ & 0.11 & & \\
\hline IgM level & & & & & & & $0.12(0.10-1.50)$ & 0.13 \\
\hline
\end{tabular}

Bold values indicate statistical significance

CI confidence intervals, EDSS Expanded Disability Status Scale, OR odds ratio

of confirmed antimicrobials is listed in the ESM). This represented a higher rate of antibiotic prescription than previously published data for rituximab, a chimeric anti-CD20 monoclonal antibody, in a national register [9], although it should be noted our cohort was significantly smaller in size. Similarly, the higher rate of serious infection seen in our study compared with the OPERA-OLE [20] study likewise needs to be interpreted with caution, given the low number of serious infections (5).

The association of younger age with risk of infection and antimicrobial use was an unexpected finding. Of patients younger than the median age of 42.58 (data not shown), $52.7 \%$ (48 of 91) reported an infection, compared with $43.64 \%$ (41 of 94 ) in those at this age or greater. In the younger group, $34.1 \%$ of patients ( 31 of 91 ) reported an upper respiratory tract infection, whereas in the older group, $21.3 \%$ of patients (20 of 94 ) reported an upper respiratory tract infection, accounting for the largest proportion of the difference in total frequency of infections between these groups. Urinary tract infections, occurring in $13.2 \%$ (12 of 91) and 10.6\% (10 of 94) of patients in the younger and older age groups, respectively, were more evenly balanced. The higher rate of infection (and particularly upper respiratory tract infections) reported in younger patients we felt is unlikely attributable exclusively to age, but rather, may have been influenced by factors such as exposure to young children, and older patients potentially isolating more stringently given public awareness of the heightened risk for severe COVID-19 with increasing age. A differential effect of recall bias cannot be excluded.

The association of antimicrobial use with increasing disease duration seen in our study represents a novel finding. Greater cumulative exposure to DMTs with longer disease duration, and greater likelihood of having medical comorbidities may underpin this relationship. Cardiac and pulmonary disease, for instance, have been associated with increased risk of severe infection in rituximab-treated patients [21]. Indeed, in the era of COVID-19, medical comorbidities are associated with greater COVID-19 severity in the general population $[22,23]$ and patients with MS [23-25]; it thus follows that patients with CD20 depletion and medical comorbidities may have a heightened risk of infection compared with those without.

Increasing age and EDSS score are likewise associated with greater COVID-19 severity in patients with MS [24, 25], and along with disease duration, are more broadly associated with risk of infection-related hospitalisation in MS patients [26]. In our study, consistent with these findings, increasing EDSS was associated with an increased risk of antimicrobial use. Higher EDSS is associated with more frequent urological complications, including infection [27], with lower urinary tract dysfunction, catheterisation, and greater functional dependence leading to compromised hygiene, all potentially increasing the risk of urinary tract infections [27]. Likewise, respiratory dysfunction is associated with higher EDSS in patients with MS [28], predisposing to aspiration pneumonia and lung infections [29].

No relationship between prior immunosuppression and infection or antimicrobial use was seen in our study, but given the heterogeneous levels of immune suppression associated with these agents as a group, variable wash-out periods and variable subsequent duration of ocrelizumab use, a more meaningful analysis was precluded. Future studies could explore the differential effect of prior DMTs on risk of infection in ocrelizumab-treated patients with larger numbers of patients in respective groups.

In our cohort, given only small proportions of progressive phenotypes-with 14 patients in total (7.6\%) with either PPMS or SPMS - the impact of MS course on infection risk could not be fully explored, though an association between 
higher EDSS and antimicrobial use was seen. In the phase III RCT, serious infections occurred in a similar number of patients receiving ocrelizumab as compared with placebo [16] and remained balanced in the OLE [30]. Conversely, in rituximab-treated PPMS patients, in the phase II/III RCT, serious infections occurred compared with in those receiving placebo. This disparity may reflect a higher mean age and EDSS in PPMS populations than is typically seen in RRMS [31].

The association of lower serum IgG levels and risk of infection as seen in our study is consistent with findings from phase III ocrelizumab OLE data, with significant associations of $\operatorname{IgG}$ and $\operatorname{IgM}$ but not IgA levels below the lower limit of normal and serious infections observed [32]. Rituximab-related hypogammaglobulinaemia in neuromyelitis optica spectrum disorder is similarly associated with serious infection [33]. Conversely, in an MS cohort treated with ocrelizumab [34], neither IgG nor IgM predicted the risk of infection during a 26-month median follow-up. A study of rituximab-treated rheumatoid arthritis patients with concurrent methotrexate similarly demonstrated that rates of serious infection did not differ before and after the development of $\operatorname{IgG}$ or IgM below the lower limit of normal, though the minimum follow-up was 4 months [35]. These findings may underscore the need for longer follow-up to better delineate the consequence of B-cell depletion mediated hypogammaglobulinaemia on infection risk. Indeed, OLE data for ocrelizumab in RRMS [20] and PPMS [30] demonstrated similar increases in the proportions of patients developing $\operatorname{IgG}, \operatorname{Ig} \mathrm{A}$ and $\operatorname{IgM}$ below the lower limit of normal over time, consistent with data in rheumatological [35, 36] and other diseases [37] in demonstrating a relationship between the number of doses of anti-CD20 therapy and risk of hypogammaglobulinaemia.

The association of lower serum $\operatorname{Ig} \mathrm{A}$ in our study and the risk of infection represents an uncommonly reported finding. Reduced serum IgA in the context of B-cell depletion has generally not been considered as a risk factor for infection [38]. The majority of patients with primary $\operatorname{IgA}$ deficiency are asymptomatic, though some patients have a propensity to recurrent sinopulmonary infections [39]. Lower serum Ig A levels may be a surrogate marker of underlying immune perturbation, indirectly contributing to infection risk. The reduction of serum $\operatorname{IgG}$ and $\operatorname{IgA}$ post B-cell depletion remains incompletely understood, given long-lived plasma cells, which do not express CD20 [40], appear to be impervious to CD20-depleting therapy [41]. Mechanisms may include a reduced pool of memory B-cells to differentiate into plasmablasts and plasma cells [42]. This, however, could only partly explain it, given that, in mouse models, memory B-cells are not required to maintain bone marrow plasma cells $[41,43]$. The more common occurrence of IgM hypogammaglobulinaemia following treatment with anti-CD20 therapy may likewise reflect a dependence of IgM-secreting plasma cells on a pool of memory B-cells, though this in itself may be incompletely understood, given the presence of long-lived IgM plasma cells [44]. In addition to clinical significance, mechanisms of anti-CD20-related hypogammaglobulinaemia also require future elucidation.

Furthermore, beyond the scope of our study, owing to a lack of available data, multiple studies have implicated pre-CD20 therapy hypogammaglobulinaemia as a risk factor for future infections $[21,35]$, fostering the recommendation to assess patients' immunoglobulin levels prior to CD20 therapy initiation, which is often not done in clinical practice [37].

No association was seen in our study between total lymphocyte count and lymphocyte subset levels and risk of self-reported infection or antimicrobial use. Considering $\mathrm{CD} 19^{+}$levels, given the profoundly reduced mean count in our cohort, likely due to the majority of patients having undetectable levels, an association between B-cell depletion and infection risk, even if present, could not be borne out. In ocrelizumab-treated patients with PPMS [16], a reduction in $\mathrm{CD}^{+} \mathrm{T}$ cells and an initial decrease in $\mathrm{CD}^{+}$cells have been demonstrated. In patients with MS, both rituximab [45] and ocrelizumab [46] have been shown to deplete CD20 T-cells, which comprise a minority of overall T-cells, and are thought to represent a highly activated T-cell population with pro-inflammatory properties [46]. Nonetheless, the significance of these findings requires further elaboration, and the effect of $\mathrm{CD} 3{ }^{+} \mathrm{CD} 20^{+}$depletion in the context of $\mathrm{CD} 20^{+}$depletion, if any, on risk of infection in clinical practice remains unknown.

Similarly, no relationship was seen between neutrophil counts and self-reported infections or anti-microbial use. In part, this may be reflective of the generally robust absolute neutrophil counts (ANC) in our study, with only two patients having a grade 1 neutropaenia (data not shown). In the phase III PPMS ocrelizumab study, $1 \%$ of patients developed neutrophil counts $<1.0 \times 10^{9} / \mathrm{L}$, without associated infections [47]. Furthermore, consistent with the rare association in relation to rituximab therapy, late-onset neutropaenia, described as an ANC below $1.5 \times 10^{9 /} \mathrm{L},>4$ weeks after the last dose of therapy [48], has been reported in MS patients receiving ocrelizumab, without any infectious complications [49-51]. Given the rarity of anti-CD20-related neutropaenia, larger studies with longer follow-up would be of interest to explore the incidence, infective risks and management implications.

During the study period, three patients discontinued ocrelizumab. One was a patient hospitalised with a diagnosis of a viral infection with superimposed bacterial infection, who had been deemed to have experienced frequent infections longitudinally whilst receiving ocrelizumab; another was a patient admitted with osteomyelitis, and in another 
patient, ocrelizumab was discontinued due to subclinical IgG hypogammaglobulinaemia. Historical patients in our institution to have discontinued ocrelizumab prior to our study period were excluded to avoid increased recall bias in assessing infections not reflected in medical records.

Given the low proportion of patients who were current smokers (15.1\%), and the potential impact of COVID-19 public health measures on rates of influenza transmission, smoking and influenza vaccination were not considered in our analyses as potential factors modulating infection risk. Of seven patients reporting an influenza-like illness, three had received the seasonal influenza vaccine. None of the patients who received the pneumococcal vaccine reported symptoms suggestive of lower respiratory tract infections.

Notwithstanding the reported infections, reassuringly, only $16 \%$ of patients reported an increase in the frequency of infections sustained whilst receiving ocrelizumab. Further studies are required to evaluate the head-to-head difference in rate of infection in ocrelizumab-treated patients and those on other DMTs, particularly other higher efficacy DMTs.

Our work adds to the body of literature detailing realworld outcomes in MS patients treated with ocrelizumab and safety outcomes. In comparison with further previously published real-world series [52-56], our reported frequency of infections was significantly higher; although, with the exception of the incorporation of phone interviews in one study [52], these studies largely included infections as they were recorded in medical records. Notwithstanding the possibility of greater accuracy in defining infections, a review of medical records alone may under-report the true incidence, particularly for milder infections, which are less likely to be raised in standard outpatient consultations. Interestingly, few infection-related hospitalisations were reported; although, similar to our study, studies were limited to the initial cycles of ocrelizumab treatment, with a median of three infusions in two studies [55,56], and a median follow-up period of 200 days after initiation in another study [53]. Thus, particularly considering the increasing incidence of hypogammaglobulinaemia with longer treatment duration, the need for longer-term data remains. Indeed, a large, prospective realworld study evaluating safety outcomes, including infection, in ocrelizumab-treated MS patients is currently under way [57]. Further, where assessed, in one study, a higher rate of adverse events were seen in patients previously treated with DMTs, though this was not statistically significant, and the absolute numbers of infections were low [56]. Beyond this, authors generally concluded that there were no relationships between baseline demographics and infection risk [52, 55], and though an overall and pre- and post-treatment decline in IgG was seen in one study, conclusions couldn't be drawn as to the relationship of these findings with risk of infection [52]. Finally, in a comparison of the tolerability and immune effects of ocrelizumab and rituximab in MS [58], reductions in IgG levels in ocrelizumab-treated patients and IgM levels in both groups were seen, but the significance of these findings with respect to infection was not assessed.

Our study has a number of limitations. The retrospective manner of data collection raises the possibility of recall and reporting bias, with the possibility that reports of infection and antimicrobial use could be either over- or under-reported. Further, it is possible patient-completed and phone-completed surveys may have been associated with varying degrees of recall bias. Given the pre-defined descriptions of infection, an accurate and proven diagnosis of a given type of infection was not possible. Infections being defined via self-reported survey prevents comparison of our findings with studies reporting infection based on a clinical assessment and diagnosis. Further, our study evaluated patients at a single time point, focusing on the rates of infection 'since' their last infusion. Longer duration followup may have provided a better reflection of possible associations between clinical factors and infection risk, but at risk of further increasing recall bias. A lack of centralised healthcare records meant it was not possible to verify all infections where a patient had a medical assessment, as patients would often attend a different general practice to that listed in hospital records or may attend multiple practices. Considering antimicrobial usage, limiting this variable to those in whom antimicrobial use could be confirmed by review of medical records alone was deemed to significantly underestimate the true proportion of such patients, given the aforementioned difficulty in accessing all relevant records.

Due to COVID-19 public health measures such maskwearing in public, restrictions upon leaving one's home and social distancing, the frequency of airborne and droplettransmitted respiratory infections may have been reduced for a significant proportion of our study. Also, we were not able to account for patients' medical comorbidities, which may in turn have independent associations with risk of infection. Laboratory measurements in our cohort were obtained from multiple laboratories, which could have influenced immunoglobulin and lymphocyte results, and measurements were not consistently available for each patient, as previously detailed.

Finally, due to the limited sample size, clinically meaningful associations may have been missed in the analyses. We were only able to assess linear relationships between the predictors and the outcomes.

\section{Conclusions}

Overall, our study demonstrated that in a large cohort of MS patients receiving ocrelizumab, higher serum IgG and IgA levels and older age were associated with a reduced odds of infection. Our study also showed that older age and higher 
EDSS were associated with reduced odds of antimicrobial use, and longer disease duration and higher EDSS were associated with an increased odds of antimicrobial use. Although some of these factors are well described predicators, serum IgA levels and risk of infection in ocrelizumab-treated patients is a novel finding. Our results highlight the need to monitor patients' immunoglobulin profiles prior to commencing ocrelizumab and during the maintenance course. Further studies are required to delineate the relationship of immunoglobulin levels and infection risk, and more broadly, assess long-term risks factors for infection in patients receiving ocrelizumab.

Supplementary Information The online version contains supplementary material available at https://doi.org/10.1007/s40263-021-00810-3.

\section{Declarations}

Funding The study received no financial support.

Conflict of interest NS has received conference fee sponsorship from Roche. TK served on scientific advisory boards for Roche, Celgene, Sanofi-Genzyme, Novartis, Merck and Biogen; a steering committee for Brain Atrophy Initiative by Sanofi-Genzyme; received conference travel support and/or speaker honoraria from WebMD Global, Novartis, Biogen, Sanofi-Genzyme, Teva, BioCSL and Merck and received research support from Biogen. MMo has served on advisory board for Merck, and has received speaker honoraria from Merck and Biogen. Her institution receives funding from Merck, Australian National Health Medical Research Council, Brain Foundation, Charles and Sylvia Viertel Foundation, and MS Research Australia. The other authors declare no conflicts of interest.

Ethical approval This study was approved by The Royal Melbourne Hospital Human Research Ethics Committee (QA2020045).

Consent to participate Informed consent was obtained from each participant.

Availability of data and material Datasets acquired and analysed during the study are available from the corresponding author on request.

Author contributions Design/conceptualisation of the study: MMo, NS. Analysis/data interpretation: SS, NS, MMo, TK. Drafting and revising the manuscript: NS, MMo, VL, TK, SS. All authors give final approval of the manuscript.

\section{References}

1. Harding K, Williams O, Willis M, Hrastelj J, Rimmer A, Joseph F, et al. Clinical outcomes of escalation vs early intensive diseasemodifying therapy in patients with multiple sclerosis. JAMA Neurol. 2019;76(5):536-41.

2. Buron MD, Chalmer TA, Sellebjerg F, Barzinji I, Christensen JR, Christensen MK, et al. Initial high-efficacy disease-modifying therapy in multiple sclerosis: a nationwide cohort study. Neurology. 2020;95(8):e1041-51.

3. Brown JWL, Coles A, Horakova D, Havrdova E, Izquierdo G, Prat A, et al. Association of initial disease-modifying therapy with later conversion to secondary progressive multiple sclerosis. JAMA. 2019;321(2):175-87.

4. Persson R, Lee S, Ulcickas YM, Wagner UMCM, Minton N, Niemcryk $S$, et al. Infections in patients diagnosed with multiple sclerosis: a multi-database study. Mult Scler Relat Disord. 2020;41:101982.

5. Wijnands JM, Kingwell E, Zhu F, Zhao Y, Fisk JD, Evans C, et al. Infection-related health care utilization among people with and without multiple sclerosis. Mult Scler. 2017;23(11):1506-16.

6. Marrie RA, Elliott L, Marriott J, Cossoy M, Blanchard J, Leung $\mathrm{S}$, et al. Effect of comorbidity on mortality in multiple sclerosis. Neurology. 2015;85(3):240-7.

7. Harding K, Zhu F, Alotaibi M, Duggan T, Tremlett H, Kingwell E. Multiple cause of death analysis in multiple sclerosis: a population-based study. Neurology. 2020;94(8):e820-9.

8. Burkill S, Montgomery S, Hajiebrahimi M, Hillert J, Olsson T, Bahmanyar S. Mortality trends for multiple sclerosis patients in Sweden from 1968 to 2012. Neurology. 2017;89(6):555-62.

9. Luna G, Alping P, Burman J, Fink K, Fogdell-Hahn A, Gunnarsson $\mathrm{M}$, et al. Infection risks among patients with multiple sclerosis treated with fingolimod, natalizumab, rituximab, and injectable therapies. JAMA Neurol. 2020;77(2):184-91.

10. Wijnands JMA, Zhu F, Kingwell E, Fisk JD, Evans C, Marrie RA, et al. Disease-modifying drugs for multiple sclerosis and infection risk: a cohort study. J Neurol Neurosurg Psychiatry. 2018;89(10):1050-6.

11. Franciotta D, Salvetti M, Lolli F, Serafini B, Aloisi F. B cells and multiple sclerosis. Lancet Neurol. 2008;7(9):852-8.

12. Sabatino JJ, Pröbstel AK, Zamvil SS. B cells in autoimmune and neurodegenerative central nervous system diseases. Nat Rev Neurosci. 2019;20(12):728-45.

13. Hauser SL, Waubant E, Arnold DL, Vollmer T, Antel J, Fox RJ, et al. B-cell depletion with rituximab in relapsing-remitting multiple sclerosis. N Engl J Med. 2008;358(7):676-88.

14. Hauser SL, Bar-Or A, Comi G, Giovannoni G, Hartung HP, Hemmer B, et al. Ocrelizumab versus interferon Beta-1a in relapsing multiple sclerosis. N Engl J Med. 2017;376(3):221-34.

15. Hauser SL, Bar-Or A, Cohen JA, Comi G, Correale J, Coyle PK, et al. Ofatumumab versus teriflunomide in multiple sclerosis. $\mathrm{N}$ Engl J Med. 2020;383(6):546-57.

16. Montalban X, Hauser SL, Kappos L, Arnold DL, Bar-Or A, Comi $\mathrm{G}$, et al. Ocrelizumab versus placebo in primary progressive multiple sclerosis. N Engl J Med. 2017;376(3):209-20.

17. Emery P, Rigby W, Tak PP, Dörner T, Olech E, Martin C, et al. Safety with ocrelizumab in rheumatoid arthritis: results from the ocrelizumab phase III program. PLoS ONE. 2014;9(2):e87379.

18. Sormani MP, De Rossi N, Schiavetti I, Carmisciano L, Cordioli C, Moiola L, et al. Disease-modifying therapies and coronavirus disease 2019 severity in multiple sclerosis. Ann Neurol. 2021;89(4): 780-789.

19. Simpson-Yap S. First results of the COVID-19 in MS global data sharing initiative suggest antiCD20 DMTs are associated with worse COVID-19 outcomes. MS Virtual 2020;2020:SS02.04.

20. Hauser SL, Kappos L, Arnold DL, Bar-Or A, Brochet B, Naismith RT, et al. Five years of ocrelizumab in relapsing multiple sclerosis: OPERA studies open-label extension. Neurology. 2020;95(13):e1854-67.

21. Gottenberg JE, Ravaud P, Bardin T, Cacoub P, Cantagrel A, Combe B, et al. Risk factors for severe infections in patients with rheumatoid arthritis treated with rituximab in the autoimmunity and rituximab registry. Arthritis Rheum. 2010;62(9):2625-32.

22. Guan WJ, Liang WH, Zhao Y, Liang HR, Chen ZS, Li YM, et al. Comorbidity and its impact on 1590 patients with COVID-19 in China: a nationwide analysis. Eur Respir J. 2020;55(5):1-56.

23. Petrilli CM, Jones SA, Yang J, Rajagopalan H, O'Donnell L, Chernyak Y, et al. Factors associated with hospital admission 
and critical illness among 5279 people with coronavirus disease 2019 in New York City: prospective cohort study. BMJ. 2020;369:m1966.

24. Parrotta E, Kister I, Charvet L, Sammarco C, Saha V, Charlson RE, et al. COVID-19 outcomes in MS: observational study of early experience from NYU Multiple Sclerosis Comprehensive Care Center. Neurol Neuroimmunol Neuroinflamm. 2020;7(5):1-9.

25. Louapre C, Collongues N, Stankoff B, Giannesini C, Papeix C, Bensa $\mathrm{C}$, et al. Clinical characteristics and outcomes in patients with Coronavirus disease 2019 and multiple sclerosis. JAMA Neurol. 2020;77(9):1079-88.

26. Pirttisalo AL, Sipilä JOT, Viitala M, Soilu-Hänninen M. Trends and characteristics of infection-related hospital admissions in multiple sclerosis patients in Southwest Finland in 2009-2018. Mult Scler Relat Disord. 2020;44:102328.

27. Abello A, Badin J, Das AK. Worsening disability status in multiple sclerosis predicts urologic complications. Int Urol Nephrol. 2020;52(5):859-63.

28. Buyse B, Demedts M, Meekers J, Vandegaer L, Rochette F, Kerkhofs L. Respiratory dysfunction in multiple sclerosis: a prospective analysis of 60 patients. Eur Respir J. 1997;10(1):139-45.

29. Tzelepis GE, McCool FD. Respiratory dysfunction in multiple sclerosis. Respir Med. 2015;109(6):671-9.

30. Wolinsky JS, Arnold DL, Brochet B, Hartung HP, Montalban X, Naismith RT, et al. Long-term follow-up from the ORATORIO trial of ocrelizumab for primary progressive multiple sclerosis: a post-hoc analysis from the ongoing open-label extension of the randomised, placebo-controlled, phase 3 trial. Lancet Neurol. 2020;19(12):998-1009.

31. Hawker K, O'Connor P, Freedman MS, Calabresi PA, Antel J, Simon $\mathrm{J}$, et al. Rituximab in patients with primary progressive multiple sclerosis: results of a randomized double-blind placebocontrolled multicenter trial. Ann Neurol. 2009;66(4):460-71.

32. Derfuss T. Serum immunoglobulin levels and risk of serious infections in the pivotal phase III trials of ocrelizumab in multiple sclerosis and their open-label extensions. ECTRIMS Online Library; 2019.

33. Tallantyre EC, Whittam DH, Jolles S, Paling D, Constantinesecu C, Robertson NP, et al. Secondary antibody deficiency: a complication of anti-CD20 therapy for neuroinflammation. J Neurol. 2018;265(5):1115-22.

34. Smoot K. The impact of ocrelizumab on immunoglobulin levels and the risk of infection. In: Moreno C, Stuchiner T, Chen C, Cohan S, editors. ECTRIMS Online Library; 2019.

35. van Vollenhoven RF, Fleischmann RM, Furst DE, Lacey S, Lehane PB. Longterm safety of rituximab: final report of the rheumatoid arthritis global clinical trial program over 11 years. J Rheumatol. 2015;42(10):1761-6.

36. De La Torre I, Leandro MJ, Valor L, Becerra E, Edwards JC, Cambridge G. Total serum immunoglobulin levels in patients with RA after multiple B-cell depletion cycles based on rituximab: relationship with B-cell kinetics. Rheumatology (Oxford). 2012;51(5):833-40.

37. Barmettler S, Ong MS, Farmer JR, Choi H, Walter J. Association of Immunoglobulin levels, infectious risk, and mortality with rituximab and hypogammaglobulinemia. JAMA Netw Open. 2018;1(7):e184169.

38. Kridin K, Ahmed AR. Post-rituximab immunoglobulin M (IgM) hypogammaglobulinemia. Autoimmun Rev. 2020;19(3):102466.

39. Yel L. Selective IgA deficiency. J Clin Immunol. 2010;30(1):10-6.

40. Halliley JL, Tipton CM, Liesveld J, Rosenberg AF, Darce J, Gregoretti IV, et al. Long-lived plasma cells are contained within the CD19(-)CD38(hi)CD138(+) subset in human bone marrow. Immunity. 2015;43(1):132-45.

41. DiLillo DJ, Hamaguchi Y, Ueda Y, Yang K, Uchida J, Haas $\mathrm{KM}$, et al. Maintenance of long-lived plasma cells and serological memory despite mature and memory B cell depletion during CD20 immunotherapy in mice. J Immunol. 2008;180(1):361-71.

42. Christou EAA, Giardino G, Worth A, Ladomenou F. Risk factors predisposing to the development of hypogammaglobulinemia and infections post-Rituximab. Int Rev Immunol. 2017;36(6):352-9.

43. Ahuja A, Anderson SM, Khalil A, Shlomchik MJ. Maintenance of the plasma cell pool is independent of memory B cells. Proc Natl Acad Sci USA. 2008;105(12):4802-7.

44. Bohannon C, Powers R, Satyabhama L, Cui A, Tipton C, Michaeli $\mathrm{M}$, et al. Long-lived antigen-induced IgM plasma cells demonstrate somatic mutations and contribute to long-term protection. Nat Commun. 2016;06(7):11826.

45. Palanichamy A, Jahn S, Nickles D, Derstine M, Abounasr A, Hauser SL, et al. Rituximab efficiently depletes increased CD20expressing $\mathrm{T}$ cells in multiple sclerosis patients. J Immunol. 2014;193(2):580-6.

46. Gingele S, Jacobus TL, Konen FF, Hümmert MW, Sühs KW, Schwenkenbecher P, et al. Ocrelizumab depletes CD20 ${ }^{+} \mathrm{T}$ cells in multiple sclerosis patients. Cells. 2018;8(1):1-6.

47. Ocrevus Prescribing Information, accessed from https://www. gene.com/download/pdf/ocrevus_prescribing.pdf. 2020 Dec version. Accessed 7 Mar 2021.

48. Tesfa D, Palmblad J. Late-onset neutropenia following rituximab therapy: incidence, clinical features and possible mechanisms. Expert Rev Hematol. 2011;4(6):619-25.

49. Cohen BA. Late-onset neutropenia following ocrelizumab therapy for multiple sclerosis. Neurology. 2019;92(9):435-6.

50. Zanetta C, Robotti M, Nozzolillo A, Sangalli F, Liberatore G, Nobile-Orazio E, et al. Late onset absolute neutropenia associated with ocrelizumab treatment in multiple sclerosis: a case report and review of the literature. J Neurol Sci. 2020;02(409):116603.

51. Auer M, Bsteh G, Hegen H, Wurth S, Zinganell A, Berger T, et al. Late-onset neutropenia in a multiple sclerosis patient after first dose ocrelizumab switched from rituximab. Mult Scler Relat Disord. 2020;43:102155

52. Prockl V, Nickel FT, Utz KS, Fröhlich K, Engelhorn T, Hilz MJ, et al. Real world application of ocrelizumab in multiple sclerosis: Single-center experience of 128 patients. J Neurol Sci. 2020;08(415):116973.

53. Ellwardt E, Rolfes L, Klein J, Pape K, Ruck T, Wiendl H, et al. Ocrelizumab initiation in patients with MS: a multicenter observational study. Neurol Neuroimmunol Neuroinflamm. 2020;7(4):1-8.

54. Rauer S, Hoshi MM, Pul R, Wahl M, Schwab M, Haas J, et al. Ocrelizumab treatment in patients with primary progressive multiple sclerosis: short-term safety results from a compassionate use Programme in Germany. Clin Neurol Neurosurg. 2020;197:106142.

55. Fernandez-Diaz E, Perez-Vicente JA, Villaverde-Gonzalez R, Berenguer-Ruiz L, Candeliere Merlicco A, Martinez-Navarro ML, et al. Real-world experience of ocrelizumab in multiple sclerosis in a Spanish population. Ann Clin Transl Neurol. 2021;8(2):385-94.

56. Sempere AP, Berenguer-Ruiz L, Borrego-Soriano I, Burgos-San Jose A, Concepcion-Aramendia L, Volar L, et al. Ocrelizumab in multiple sclerosis: a real-world study from Spain. Front Neurol. 2020;11:592304.

57. Dirks P, Zingler V, Leemhuis J, Berthold H, Hieke-Schulz S, Wormser D, et al. Design of a non-interventional post-marketing study to assess the long-term safety and effectiveness of 
ocrelizumab in German real world multiple sclerosis cohorts- the CONFIDENCE study protocol. BMC Neurol. 2020;20(1):95.

58. Evertsson B, Hoyt T, Christensen A, Nimer FA, Foley J, Piehl F. A comparative study of tolerability and effects on immunoglobulin levels and CD19 cell counts with ocrelizumab vs low dose of rituximab in multiple sclerosis. Mult Scler J Exp Transl Clin. 2020;6(4):2055217320964505.

\section{Authors and Affiliations}

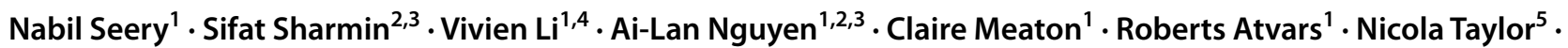
Kelsey Tunnell ${ }^{5}$ John Carey ${ }^{5}$. Mark P. Marriott ${ }^{1,6} \cdot$ Katherine A. Buzzard $^{1,6} \cdot$ Izanne Roos $^{1,2,3} \cdot$ Chris Dwyer $^{1,4}$. Josephine Baker $^{1} \cdot$ Lisa Taylor $^{1} \cdot$ Kymble Spriggs $^{3,7} \cdot$ Trevor J. Kilpatrick $^{1,4} \cdot$ Tomas Kalincik $^{1,2,3} \cdot$ Mastura Monif $^{1,8,9}$

1 Department of Neurology, Melbourne MS Centre, Royal Melbourne Hospital, Parkville, VIC 3050, Australia

2 Clinical Outcomes Research Unit, University of Melbourne, Melbourne, VIC 3010, Australia

3 Department of Medicine, University of Melbourne, Melbourne, VIC 3010, Australia

4 Florey Institute of Neuroscience and Mental Health, University of Melbourne, Parkville, VIC 3052, Australia

5 Day Medical Centre, Royal Melbourne Hospital, Parkville, VIC 3050, Australia
6 Department of Neuroscience, Eastern Health Clinical School, Eastern Health, Box Hill, VIC 3128, Australia

7 Department of Immunology, The Royal Melbourne Hospital, Parkville, VIC 3050, Australia

8 Department of Neuroscience, Monash University, Melbourne, VIC 3004, Australia

9 MS and Neuroimmunology Department, Alfred Hospital, Melbourne, VIC 3004, Australia 